\title{
Kinetic study of the ion passage over the solar wind MHD termination shock
}

\author{
H.-J. Fahr and M. Siewert
}

\begin{abstract}
Institut für Astrophysik und Extraterrestrische Forschung (now part of the Argelander Institut für Astronomie) der Universität Bonn, Auf dem Huegel 71, 53121 Bonn, Germany

e-mail: msiewert@astro.uni-bonn.de
\end{abstract}

Recieved 4 May 2006 / Accepted 30 June 2006

\section{ABSTRACT}

\begin{abstract}
Aims. We study the classical problem of the transition of a pressure-anisotropic plasma over a hydrodynamical shock using a purely Boltzmann-kinetical treatment.

Methods. We derive the Boltzmann equation for a plasma crossing a shock at a random orientation and evaluate the velocity space integral of the distribution function over the shock analytically. We then try to reproduce the classical MHD behavior and to evaluate the pressures parallel and perpendicular to the magnetic field by integrating the resulting Boltzmann equation over velocity space. Results. We obtain, for the first time, a completely analytical result for the downstream pressure anisotropy in the case of a perpendicular shock, closing the set of MHD jump conditions. For the parallel shock, it turns out that a single-fluid description without relaxation terms is not sufficient to describe the shock, no matter how much we fine-tune our external parameters. All these results are completely independent of the fine structure of the shock and the upstream distribution function.
\end{abstract}

Key words. plasmas - shock waves - solar wind - magnetohydrodynamics (MHD)

\section{Introduction}

As is well known from the literature, the solar wind termination shock, and in most cases also other astrophysical shocks, are theoretically well described by the application of hydrodynamical or magnetohydrodynamical fluid concepts (for more recent different model approaches to the solar wind termination shock see Zank et al. 1993; Chalov \& Fahr 1994, 1995, 1996; Zank 1999; Fahr \& Scherer 2005). For purely hydrodynamical fluids the socalled Rankine-Hugoniot relations are developed respecting the usual physical conservation laws for the mass, the momentum, the energy, and the entropy flows. Usually they appear in the form given by, e.g., Serrin (1959), Zel'dovich \& Raizer (1966) or Landau \& Lifshitz (1977). If the plasma is magnetized and magnetic pressures and tensions cannot be neglected, then these relations have to be enlarged by corresponding magnetic terms and, under the simplifying assumption of scalar ion/electron pressures, one in that case is lead to a system of MHD shock relations given in e.g. Baumjohann \& Treumann (1996), Gombosi (1998) or Diver (2001).

The outstanding problem in these approaches is that most of the astrophysical shocks are classified by their nature as collisionless shocks, i.e., the randomization or thermalization of particle kinetic energies on the upstream side at the passage over the small scales of the shock transition (that is of the order of the ion inertial length or a proton skin depth) cannot be achieved by usual binary particle collisions. Hereby the ion inertial length, or the proton skin depth, is given by $D \simeq c / \omega_{\mathrm{pi}}$ with $\omega_{\mathrm{pi}}$ being the ion plasma frequency. So-called "collisionless shocks" establish themselves when collsionless plasmas counterstream to each other with super-alfvénic velocities or encounter an obstacle. Different types of such shocks appear in the heliosphere in the form of solar wind bowshocks ahead of cometary or planetary objects, shocks in-between corotating interaction regions, travelling interplanetary shocks, or the heliospheric termination shock. The problem, however, with these types of shocks is that they do not permit a hydrodynamical or magnetohydrodynamical treatment, at least not based on the lowest velocity moments of the distribution function, i.e., the density, the bulk velocity, and the scalar pressure. Nevertheless HD approaches based on low-order velocity moments of the distribution function are most often used to describe these shocks, though it is not explained in these approaches how the dynamic bulk energy of the upstream ions is irreversibly converted into thermal energy of the downstream ions. It is tacitly understood in the scientific community that this entropisation of the plasma flow is achieved by collective wave-particle interaction processes, which require upstream and downstream wave turbulences, not consistently included in the usual MHD approach.

General weaknesses of the MHD approach have been extensively discussed in the Proceedings of the COSPAR Colloquium "Physics of Collisionless Shocks" (Russell 1995) and in a recent review by Lembege et al. (2004). Unsolved questions concerning the behavior and the role of electrons at the shock passage and of shock-reflected ions that could be candidates to drive upstream MHD wave turbulences supporting nonlinear waveparticle dissipation processes, which are especially important. These counterflowing ion populations that cannot be described in the frame of a fluid theory where kinetic two-stream- or Buneman-instabilities are responsible for a viscous heating of the electron fluid. Different from fluid concepts, a local description of the dissipation via reflected ions needs kinetic aspects to be taken into account. The downstream concentration of the magnetic field and of the density self-consistently produces the electric field of the shock ramp that decelerates most of the ions, but also reflects a fraction of the inflowing ions, thus moving 
opposite to the inflowing upstream plasma. At present, the only hope to bring this complicated kinetic particle-field system to at least some degree of conistency is connected with shock simulation calculations within hybrid or full particle codes (e.g., see Hada et al. 2003; Scholer et al. 2003) Yet even these computationally highly pretentious simulation runs most often do not offer a stationary solution for the shock profile, but due to the accumulation of spectuularly reflected ions upstream of the shock cause the formation and repeated reformation of ramp shocks on timescales of gyroperiods of the ramp magnetic fields. While for unrealistic ion/electron mass ratios (i.e., $m_{\mathrm{i}} / m_{\mathrm{e}}=60$ ) the average shock profile attains a relatively regular shape, for realistic ratios $m_{\mathrm{i}} / m_{\mathrm{e}}=1840$ a completely erratic profile is found. Most unclear in all the simulation runs remains the role of shockheated electrons. Hybrid simulation codes show that they can only achieve reasonable results for unresasonably high electron resistivities (Scudder 1995), which perhaps could be ascribed to ion-acoustic instabilities or to Coulomb collision mediation, but, as could be shown in particle-particle simulations, neither ion-acoustic instabilities nor Coulomb collision effects are likely to become of the required importance (see Wilkinson 1991; Thomsen et al. 1985). Thus it may be concluded here that neither hybrid codes nor full particle in cell codes are able at present to adequately represent the full physics of collisionless shocks. We thus may try another way to study the kinetic structure of collisionless shocks starting from MHD shock solutions, which yield the basic shock structure of the plasma density, the bulk velocity, and the magnetic field, and then solve the kinetic transport equation for the ion distribution function at the ion passage over this shock structure.

\section{The Boltzmann equation}

We start from solutions of the well-known MHD shock relations, which are considered to give valid connections of the fluid and field properties of the upstream with the downstream MHD plasma flows. From these solutions we obtain the main plasma properties like the plasma density and the magnitude and direction of the plasma bulk velocity and of the frozen-in magnetic field (e.g., see Baumjohann \& Treumann 1996; Gombosi 1998; Diver 2001).

What we furthermore aim at here, is the change of the kinetic properties of the ion distribution function at the ion passage from the upstream to the downstream side of the shock. To learn about these changes we integrate the Boltzmann-Vlasow equation that kinetically describes the phase-space change of the ion distribution function $f(\vec{v}, s)$. Here $\vec{v}$ is the individual ion velocity vector, and $s$ is a streamline coordinate counted along the streamline that leads from the upstream to the downstream side. For a local planar shock, which may be considered here, this coordinate simply measures the distance in the direction of the shock normal from the shock. Neglecting in the first step an explicit local time dependence and any form of stochastic interaction processes like wave-particle interactions, the Boltzmann equation is simply

$\left(\vec{v} \cdot \nabla_{r}\right) f(\vec{v}, s)+\left(\vec{k} \cdot \nabla_{v}\right) f(\vec{v}, s)=0$,

where $\vec{k}$ comprises all locally acting forces per ion mass, and where $\nabla_{r}$ and $\nabla_{v}$ are the nabla operators differentiating with respect to space and velocity coordinates, respectively.

Now we want to make use of the knowledge of the MHD quantities and for that purpose want to transform the upper Boltzmann equation into the plasma bulk frame moving with the plasma bulk velocity $\vec{U}$. In this frame the distribution function may be denoted by $\bar{f}(\vec{w}, s)$, where $\vec{w}$ denotes the individual ion velocity in the plasma bulk frame. In this frame, no electric forces are operating since these are just compensated by the Lorentz forces $\overrightarrow{K_{L, U}}=(e / c)[\vec{U} \times \vec{B}]$. However, some additional forces are appearing in this frame, since it is accelerated with respect to the solar frame due to the change of the velocity $\vec{U}$ with the proper time $\tau$ given by $\left.\mathrm{d} \vec{U} / \mathrm{d} \tau=\overrightarrow{(U} \cdot \nabla_{r}\right) \vec{U}$.

The most general covariant 8-dimensional phase-space formulation of the Boltzmann-Vlasow equation that can be applied to that case was given in the following form (see Landau \& Lifshitz 1977):

$\breve{L}(\bar{f}(\vec{w}, s))+e \overleftrightarrow{F_{\beta}^{\alpha}} p^{\beta} \frac{\partial}{\partial p^{\alpha}} \bar{f}(\vec{w}, s)=0$

where $\overleftrightarrow{F}_{\beta}^{\alpha}$ is the electromagnetic field tensor and $\boldsymbol{L}$ is the covariant Liouville operator given by:

$\breve{L}=p^{\alpha} \frac{\partial}{\partial x^{\alpha}}-\Gamma_{\mu \nu}^{\alpha} p^{\mu} p^{\nu} \frac{\partial}{\partial p^{\alpha}}$

Here the following covariant phase-space vectors and coordinates are used:

$\vec{x}=\left\{x^{0}=c t, x^{1}, x^{2}, x^{3}\right\}$

and:

$\vec{p}=\left\{p^{0}=E / c, p^{1}, p^{2}, p^{3}\right\}$

The triple index functions $\Gamma_{\mu \nu}^{\alpha}$ are the so-called affine connections. They cannot be identified with the so-called Christoffel symbols known from general relativity to describe the metric effects of gravitational fields, since the former cannot be derived as space-time derivatives of metric tensor elements, while the latter of course can. In our case they simply describe the momentum vector transformations in an accelerated reference frame that locally moves with the plasma bulk velocity $\vec{U}$. These affine connections are explicitly developed by Webb (1985). Taking his derivations for the above triple index functions $\Gamma_{\mu \nu}^{\alpha}$ and applying to his results, the simplifying conditions given in our case here, namely that the velocity $\vec{U}$ only varies with respect to the line element $s$ measured along the normal to the shock surface, then yields the following simplified form of affine connections:

$\Gamma_{00}^{n}=\gamma^{3}(u)\left(\frac{\partial U_{n}}{\partial t}+U_{n} \frac{\partial U_{n}}{\partial s}\right)$

$\Gamma_{0 n}^{n}=\gamma^{3}(u)\left(U_{n} \frac{\partial U_{n}}{\partial t}+\frac{\partial U_{n}}{\partial s}\right)$

where $\gamma(U)=\left(1-U^{2} / c^{2}\right)^{-1 / 2}$ is the Lorentz factor. All the other functions besides the above-mentioned ones do vanish. On the basis of these facts and the fact that $\vec{U}$ does not explicitly depend on time, the Boltzmann-Vlasow equation in the locally inertial bulk frame moving with $\vec{U}$ for a non-relativistic ion population (i.e., $\gamma(U) \simeq 1, m=m_{0}, E=p^{2} / 2 m_{0}$ and $p=m_{0} w$ ) then attains the following form:

$$
\begin{aligned}
\frac{\mathrm{d}}{\mathrm{d} t} \bar{f}(\vec{w}, s)= & \frac{\partial}{\partial t} \bar{f}(\vec{w}, s)+w_{\|} \cos \alpha \frac{\mathrm{d}}{\mathrm{d} s} \bar{f}(\vec{w}, s) \\
& +\left(U_{n} \frac{\mathrm{d}}{\mathrm{d} s} \vec{U} \cdot \nabla_{w}\right) \bar{f}(\vec{w}, s)+\left\langle\left(\vec{k}_{L} \cdot \nabla_{w}\right)\right\rangle_{\tau_{g}} \bar{f}(\vec{w}, s) \\
= & 0 .
\end{aligned}
$$


The local time derivative $\partial / \partial t$ in this case only appears since it results from the convective change of the distribution function occuring in the solar rest frame and given by:

$$
\frac{\partial}{\partial t} \bar{f}(\vec{w}, s)=U_{n} \frac{\mathrm{d}}{\mathrm{d} s} \bar{f}(\vec{w}, s)
$$

and thus leads to the following Boltzmann-Vlasow equation:

$$
\begin{array}{r}
\left(U_{n}+w_{\|} \cos \alpha\right) \frac{\mathrm{d}}{\mathrm{d} s} \bar{f}(\vec{w}, s)+\left(U_{n} \frac{\mathrm{d}}{\mathrm{d} s} \vec{U} \cdot \nabla_{w}\right) \bar{f}(\vec{w}, s) \\
+\left\langle\left(\vec{k}_{L} \cdot \nabla_{w}\right)\right\rangle_{\tau_{g}} \bar{f}(\vec{w}, s)=0 .
\end{array}
$$

Here with the term in \langle\rangle$-$ brackets we consider effects of gyroperiod-averaged phase-space movements on the distribution function. These motions are due to gyroperiod averages over epicyclic ion motions around the local magnetic fields. The average ion velocity then is given by $\langle\vec{w}\rangle_{\tau_{g}}=\vec{w}_{\|}$, and its component into the direction of the shock normal is given by $w_{n}=w_{\|} \cos \alpha$, where $\alpha$ is the angle between the local field $\vec{B}$ and the normal $\vec{n}$. The expression $\left\langle\left(\vec{k}_{L} \cdot \nabla_{w}\right)\right\rangle_{\tau_{g}}$ gives the gyroperiod average of Lorentz accelerations of ions with a velocity $\vec{w}$. For perfectly frozen-in fields one can assume that these fields are comoving with the plasma bulk system.

For the homogeneous region far upstream of the termination shock, where temporal and spatial derivatives of the distribution function disappear, the actions of Lorentz forces on the distribution function also have to cancel, at least if an averaging over times large compared to the gyroperiod $\tau_{\mathrm{g}}=2 \pi / \Omega_{\mathrm{g}}=$ $2 \pi m c /(e B)$ is considered.

This explicitly means that the distribution function $\bar{f}(\vec{w})$ has to be of a form such that:

$$
\left\langle\frac{e}{m c}\left([\vec{w} \times \vec{B}] \cdot \nabla_{w}\right) \bar{f}(\vec{w})\right\rangle_{\tau_{g}}=0 .
$$

The above requirement, with the field-perpendicular velocity components given by $w_{x}=w_{\perp} \sin \phi$ and $w_{y}=w_{\perp} \cos \phi$, then transforms into:

$\left[B w_{\perp} \cos \phi\left(\vec{i}_{x} \cdot \nabla_{w}\right)-B w_{\perp} \sin \phi\left(\vec{i}_{y} \cdot \nabla_{w}\right)\right] \bar{f}(\vec{w})=0$,

where $\vec{i}_{x}$ and $\vec{i}_{y}$ are unit vectors in the $x$ - and $y$-directions of velocity space. Hereby we may assume that $\vec{i}_{x}$ is located in the plane defined by $\vec{B}$ and $\vec{n}$, while $\vec{i}_{y}$ is parallel to $[\vec{B} \times \vec{n}]$. As one can see, the above requirement is fulfilled for all values of $w$, if the velocity distribution function in the plasma bulk frame does not explicitly depend on the gyration angle $\phi$, i.e., if it is gyrotropic with $\frac{\mathrm{d}}{\mathrm{d} \phi} \bar{f}(\vec{w})=0$, because then, and only then, it holds that:

$\left(\vec{i}_{x} \cdot \nabla_{w}\right) \bar{f}(\vec{w})=\sin \varphi \frac{\mathrm{d}}{\mathrm{d} w_{\perp}} \bar{f}(\vec{w})$

and:

$\left(\vec{i}_{y} \cdot \nabla_{w}\right) \bar{f}(\vec{w})=\cos \varphi \frac{\mathrm{d}}{\mathrm{d} w_{\perp}} \bar{f}(\vec{w})$,

and as consequence of that the above relation (10) is always fulfilled.

Now we want to solve the Boltzmann equation in the plasma bulk reference frame that moves locally with the plasma bulk velocity $\vec{U}$; however, considering the inhomogeneous transition region just upstream and downstream of the shock, where $\vec{U}$ and $\vec{B}$, for a locally planar shock, are functions only of the streamline coordinate $s$. In this system the distribution function is given by $\bar{f}(\vec{w}, s)$, where the individual ion velocity $\vec{w}$ is connected with the associated velocity $\vec{v}$ in the solar rest frame by $\vec{w}=\vec{v}-\vec{U}$. Neglecting explicit time-dependences in this bulk reference system, the total time derivative of $\bar{f}(\vec{w}, s)$ thus is given by the Boltzmann equation (Eq. (8)).

The gyro-average of the Lorentz acceleration, in application to the gyrotropic distribution function $\bar{f}(\vec{w}, s)=\bar{f}\left(w_{\perp}, w_{\|}, s\right)$, explicitly assuming the conservation of the magnetic moment of each individual ion (i.e., assuming that $2 \pi / \Omega_{\mathrm{g}} \ll L / U_{n}$, where $\Omega_{\mathrm{g}}$ and $L$ are the gyrofrequency of the ion and the normal extent of the shock transition layer, respectively), can then be given by two separately contributing parts connected a) with the change of the magnitude, and $b$ ) with the change of the direction of $\vec{B}$. These two parts then lead to:

$$
\begin{aligned}
& \left\langle\left(\vec{k}_{L} \cdot \nabla_{w}\right)\right\rangle_{\tau_{g}} \bar{f}(\vec{w}, s)= \\
& \left\{\left(\left|\frac{\mathrm{d} w_{\perp}}{\mathrm{d} t}\right|_{\mu}+\left|\frac{\mathrm{d} w_{\perp}}{\mathrm{d} t}\right|_{\alpha}\right) \frac{\mathrm{d}}{\mathrm{d} w_{\perp}}+\left|\frac{\mathrm{d} w_{\|}}{\mathrm{d} t}\right|_{\alpha} \frac{\mathrm{d}}{\mathrm{d} w_{\|}}\right\} \bar{f}(\vec{w}, s) .
\end{aligned}
$$

Connected with the change of the magnetic field magnitude one derives the following term from the conservation of the magnetic particle moment $\mu=m w_{\perp}^{2} / 2 B$, i.e. from the demand $\mathrm{d} / \mathrm{d} t\left(m w_{\perp}^{2} / 2 B\right)=0$ :

$\left|\frac{\mathrm{d} w_{\perp}}{\mathrm{d} t}\right|_{\mu}=\frac{w_{\perp}}{2 B} \frac{\mathrm{d} B}{\mathrm{~d} t}=\frac{w_{\perp} U_{n}}{2 B} \frac{\mathrm{d} B}{\mathrm{~d} s}$.

Connected with the change of the magnetic field direction, one obtains in addition an apparent, or virtual, change of the velocity components due to the turn of the magnetic reference axis, as a consequence of the changing orientation of the field in the moving reference system, which is given by:

$\frac{\mathrm{d} w_{\|}}{\mathrm{d} t}=w U_{n} \frac{\mathrm{d}}{\mathrm{d} s} \cos \alpha$

and:

$\frac{\mathrm{d} w_{\perp}}{\mathrm{d} t}=w U_{n} \frac{\mathrm{d}}{\mathrm{d} s} \sin \alpha$

Here again the angle $\alpha$ denotes the inclination of $\vec{B}$ with respect to the direction of the shock normal, and $w=\sqrt{w_{\|}^{2}+w_{\perp}^{2}}$ is the magnitude of the ion velocity. Putting these things together one finds:

$$
\begin{aligned}
\left(U_{n}+w_{\|} \cos \alpha\right) & \frac{\mathrm{d}}{\mathrm{d} s} \bar{f}\left(w_{\|}, w_{\perp}, s\right)= \\
& -\left(U_{n} \frac{\mathrm{d}}{\mathrm{d} s} \vec{U}_{n} \cdot \nabla_{w}\right) \bar{f}\left(w_{\|}, w_{\perp}, s\right) \\
& -\left\{\left(\frac{w_{\perp} U_{n}}{2 B} \frac{\mathrm{d} B}{\mathrm{~d} s}+w U_{n} \frac{\mathrm{d}}{\mathrm{d} s} \sin \alpha\right) \frac{\mathrm{d}}{\mathrm{d} w_{\perp}}\right. \\
& \left.+w U_{n} \frac{\mathrm{d}}{\mathrm{d} s} \cos \alpha \frac{\mathrm{d}}{\mathrm{d} w_{\|}}\right\} \bar{f}\left(w_{\|}, w_{\perp}, s\right)
\end{aligned}
$$


which can be further developed to yield the form:

$$
\begin{aligned}
& \frac{\mathrm{d}}{\mathrm{d} s} \bar{f}\left(w_{\|}, w_{\perp}, s\right)=-\frac{U_{n}}{\left(U_{n}+w_{\|} \cos \alpha\right)} \\
& \times\left\{\frac{\mathrm{d} U_{n}}{\mathrm{~d} s}\left(\cos \alpha \frac{\mathrm{d}}{\mathrm{d} w_{\|}}+\sin \alpha\langle\sin \phi\rangle \frac{\mathrm{d}}{\mathrm{d} w_{\perp}}\right)\right. \\
& \left.+\left(\frac{w_{\perp}}{2 B} \frac{\mathrm{d} B}{\mathrm{~d} s}+w \frac{\mathrm{d}}{\mathrm{d} s} \sin \alpha\right) \frac{\mathrm{d}}{\mathrm{d} w_{\perp}}+w \frac{\mathrm{d}}{\mathrm{d} s} \cos \alpha \frac{\mathrm{d}}{\mathrm{d} w_{\|}}\right\} \bar{f}\left(w_{\|}, w_{\perp}, s\right)
\end{aligned}
$$

where $\phi$ is the gyration angle of the particles. The above can then finally be rewritten in the following more organized form:

$$
\begin{aligned}
& \frac{\mathrm{d}}{\mathrm{d} s} \bar{f}\left(w_{\|}, w_{\perp}, s\right)=-\frac{U_{n}}{\left(U_{n}+w_{\|} \cos \alpha\right)} \\
& \quad \times\left\{\left(\frac{\mathrm{d} U_{n}}{\mathrm{~d} s} \cos \alpha+w \frac{\mathrm{d}}{\mathrm{d} s} \cos \alpha\right) \frac{\mathrm{d}}{\mathrm{d} w_{\|}}+\left(\frac{\mathrm{d} U_{n}}{\mathrm{~d} s} \sin \alpha\langle\sin \varphi\rangle\right.\right. \\
& \left.\left.+\frac{w_{\perp}}{2 B} \frac{\mathrm{d} B}{\mathrm{~d} s}+w \frac{\mathrm{d}}{\mathrm{d} s} \sin \alpha\right) \frac{\mathrm{d}}{\mathrm{d} w_{\perp}}\right\} \bar{f}\left(w_{\|}, w_{\perp}, s\right) .
\end{aligned}
$$

The upper equation can still be further simplified by the fact that $\langle\sin \varphi\rangle_{\tau_{g}}=0$. Then the differential equation in principle can be integrated using a Runge-Kutta method starting from the upstream region at $s=-\epsilon$ with an initial distribution function $\bar{f}_{1}\left(w_{\|}, w_{\perp}\right)=\bar{f}\left(w_{\|}, w_{\perp},-\epsilon\right)$ and integrating over the whole extent $L$ of the shock transition layer with thickness $L=2 \epsilon$ up to $s=+\epsilon$. To do this, one has to make use of the MHD knowledge of the field and fluid quantities and their derivatives with respect to the streamline coordinate $s$.

$$
\begin{aligned}
& \frac{\mathrm{d}}{\mathrm{d} s} \bar{f}\left(w_{\|}, w_{\perp}, s\right)=-\frac{U_{n}}{\left(U_{n}+w_{\|} \cos \alpha\right)} \\
& \quad \times\left\{\left(\frac{\mathrm{d} U_{n}}{\mathrm{~d} s} \cos \alpha+w \frac{\mathrm{d}}{\mathrm{d} s} \cos \alpha\right) \frac{\mathrm{d}}{\mathrm{d} w_{\|}}\right. \\
& \left.\quad+\left(\frac{w_{\perp}}{2 B} \frac{\mathrm{d} B}{\mathrm{~d} s}+w \frac{\mathrm{d}}{\mathrm{d} s} \sin \alpha\right) \frac{\mathrm{d}}{\mathrm{d} w_{\perp}}\right\} \bar{f}\left(w_{\|}, w_{\perp}, s\right) .
\end{aligned}
$$

We note that Eq. (20) is of the form

$$
\frac{\mathrm{d}}{\mathrm{d} s} \bar{f}\left(w_{\|}, w_{\perp}, s\right)=\sum_{i=\perp, \|} a_{i}(s) \frac{\mathrm{d} b_{i}(s)}{\mathrm{d} s} \frac{\mathrm{d}}{\mathrm{d} w_{i}} \bar{f}\left(w_{\|}, w_{\perp}, s\right),
$$

where $a_{i}(s)$ and $b_{i}(s)$ are functions of MHD fluid and field quantities. The integration over the line element $s$ then yields the following solution for the distribution function downstream of the shock:

$$
\begin{aligned}
& \bar{f}_{2}\left(w_{\|}, w_{\perp}, s\right)-\bar{f}_{1}\left(w_{\|}, w_{\perp}, s\right)= \\
& \int_{-\epsilon}^{+\epsilon} \mathrm{d} s \sum_{i} a_{i}(s) \frac{\mathrm{d} b_{i}(s)}{\mathrm{d} s} \frac{\mathrm{d}}{\mathrm{d} w_{i}} \bar{f}\left(w_{\|}, w_{\perp}, s\right) .
\end{aligned}
$$

\section{Modeling of the shock transition}

To integrate Eq. (22) over the shock, it is necessary to start from the basic MHD solutions for the shock transition. Nevertheless there are two essentially different approaches possible to this problem, i.e., the shock with finite extent and the infinitesimally thin, rapid shock transition. Since the latter case is a limit of the former one, we first focus on shock with finite extent.
We assume that the extended shock ranges from $-\epsilon \leq$ $s \leq \epsilon$. For the specific form of the quantities $a_{i}$ and $b_{i}$, we chose the ansatz

$$
\begin{aligned}
& a_{i}(s)=a_{i 1}+\left(a_{i 2}-a_{i 1}\right)\left(h(s)+\frac{1}{2}\right) \\
& \frac{\mathrm{d} b_{i}(s)}{\mathrm{d} s}=\left(b_{i 2}-b_{i 1}\right) \frac{\mathrm{d} g(s)}{\mathrm{d} s} .
\end{aligned}
$$

The derivative $\mathrm{d} b_{i}(s) / \mathrm{d} s$ is nonzero only in the transition region, which naturally limits our integral to the shock transition itself. $g(s)$ and $h(s)$ are arbitrary functions that connect the upstream and the downstream regions and therefore include all details on the structure of the shock. We furthermore assume that the transition region has a similar extent for all relevant MHD quantities.

Next, we separate these functions into a symmetrical and an antisymmetrical part,

$$
\begin{aligned}
& h_{s}(s)=\frac{1}{2}(h(s)+h(-s)) \\
& h_{a}(s)=\frac{1}{2}(h(s)-h(-s)) \\
& h(s)=h_{s}(s)+h_{a}(s) .
\end{aligned}
$$

These expressions satisfy $(g, h)_{s}(s)=(g, h)_{s}(-s)$ and $(g, h)_{a}(s)=$ $-(g, h)_{a}(-s)$. Per construction, we also get $(g, h)(\epsilon)=\frac{1}{2}$, and $(g, h)(-\epsilon)=-\frac{1}{2}$, and it follows that $(g, h)_{a}(\epsilon)=(g, h)(\epsilon)=\frac{1}{2}$, $(g, h)_{s}(\epsilon)=0$ and $(g, h)_{a}(0)=0$. In addition, $d(g, h)_{a}(s) / d s$ is a symmetrical function and $d(g, h)_{s}(s) / d s$ is an antisymmetrical function. Then the integral can be evaluated without any further knowledge of $g(s)$ and $h(s)$, and we get:

$$
\begin{aligned}
& \int_{-\epsilon}^{\epsilon} a_{i}(s) \frac{\mathrm{d} b_{i}(s)}{\mathrm{d} s}= \\
& \left(b_{i 2}-b_{i 1}\right) \int_{-\epsilon}^{\epsilon}\left(a_{i 1}+\left(a_{i 2}-a_{i 1}\right)\left(h_{a}(s)+h_{s}(s)+\frac{1}{2}\right)\right) \\
& \times\left(\frac{\mathrm{d} g_{a}(s)}{\mathrm{d} s}+\frac{\mathrm{d} g_{s}(s)}{\mathrm{d} s}\right) \mathrm{d} s \\
& =\left(b_{i 2}-b_{i 1}\right) \cdot\left(\frac{1}{2}\left(a_{i 1}+a_{i 2}\right) \int_{-\epsilon}^{\epsilon} \cdot\left(\frac{\mathrm{d} g_{a}(s)}{\mathrm{d} s}+\frac{\mathrm{d} g_{s}(s)}{\mathrm{d} s}\right) \mathrm{d} s\right. \\
& \left.+\left(a_{i 2}-a_{i 1}\right) \int_{-\epsilon}^{\epsilon}\left(h_{a}(s)+h_{s}(s)\right)\left(\frac{\mathrm{d} g_{a}(s)}{\mathrm{d} s}+\frac{\mathrm{d} g_{s}(s)}{\mathrm{d} s}\right) \mathrm{d} s\right) \\
& =\frac{1}{2}\left(b_{i 2}-b_{i 1}\right)\left(a_{i 1}+a_{i 2}\right) \\
& +\left(b_{i 2}-b_{i 1}\right)\left(a_{i 2}-a_{i 1}\right) \int_{-\epsilon}^{\epsilon}\left(h_{a}(s) \frac{\mathrm{d} g_{s}(s)}{\mathrm{d} s}+h_{a}(s) \frac{\mathrm{d} g_{a}(s)}{\mathrm{d} s}\right. \\
& \left.+h_{s}(s) \frac{\mathrm{d} g_{s}(s)}{\mathrm{d} s}+h_{s}(s) \frac{\mathrm{d} g_{a}(s)}{\mathrm{d} s}\right) \mathrm{d} s \\
& =\frac{1}{2}\left(b_{i 2}-b_{i 1}\right)\left(a_{i 1}+a_{i 2}\right) \\
& +\left(b_{i 2}-b_{i 1}\right)\left(a_{i 2}-a_{i 1}\right) \int_{-\epsilon}^{\epsilon}\left(h_{a}(s) \frac{\mathrm{d} g_{s}(s)}{\mathrm{d} s}+h_{s}(s) \frac{\mathrm{d} g_{a}(s)}{\mathrm{d} s}\right) \mathrm{d} s \\
& =\frac{1}{2}\left(b_{i 2}-b_{i 1}\right)\left(a_{i 1}+a_{i 2}\right) \\
& +\frac{1}{2}\left(b_{i 2}-b_{i 1}\right)\left(a_{i 2}-a_{i 1}\right) \int_{-\epsilon}^{\epsilon}\left(h(s) \frac{\mathrm{d} g(s)}{\mathrm{d} s}-h(-s) \frac{\mathrm{d} g(-s)}{\mathrm{d} s}\right) \mathrm{d} s \\
& =\frac{1}{2}\left(b_{i 2}-b_{i 1}\right)\left(a_{i 1}+a_{i 2}\right) \text {. }
\end{aligned}
$$

From this it follows immediately that for any imaginable shock structure one is not required to know the exact structure of the 
transition region and that MHD alone does not place any constraints on the microstructure of the shock.

It is also obvious that this result even holds for an infinitesimal shock. There are other instances, though, when the thickness of the shock does matter. For example, if the shock is thicker than the average ion gyroradius, heavy ions in an astrophysical plasma will conserve their magnetic moments at the passage, while for an abrupt shock, these ions cannot adiabatically adapt to the change in the $B$-field magnitude and direction (Bzowski et al. 2006).

\section{Solving the transition equation}

Next, we use these results to evaluate Eq. (22). For the angular expressions we use

$\cos \alpha=\frac{B_{n}}{B}$

$\sin \alpha=\frac{B_{t}}{B}$,

with $B_{n}$ and $B_{t}$ as the normal and tangential magnetic field components and obtain:

$\bar{f}_{2}\left(w_{\|}, w_{\perp}\right)-\bar{f}_{1}\left(w_{\|}, w_{\perp}\right)=-\frac{1}{2}\left\{\frac{U_{n 1}}{\left(U_{n 1}+w_{\|} \cos \alpha_{1}\right)}\right.$.

$\left[\left(\frac{B_{n 1}}{B_{1}}\left(U_{n 2}-U_{n 1}\right)+w\left(\frac{B_{n 2}}{B_{2}}-\frac{B_{n 1}}{B_{1}}\right)\right) \frac{\mathrm{d} \bar{f}_{1}\left(w_{\|}, w_{\perp}\right)}{\mathrm{d} w_{\|}}\right.$

$\left.\left.+\left(\frac{w_{\perp}}{2 B_{1}}\left(B_{2}-B_{1}\right)+w\left(\frac{B_{t 2}}{B_{2}}-\frac{B_{t 1}}{B_{1}}\right)\right) \frac{\mathrm{d} \bar{f}_{1}\left(w_{\|}, w_{\perp}\right)}{\mathrm{d} w_{\perp}}\right]\right]$

$$
+\frac{U_{n 2}}{\left(U_{n 2}+w_{\|} \cos \alpha_{2}\right)} \text {. }
$$

$\left[\left(\frac{B_{n 2}}{B_{2}}\left(U_{n 2}-U_{n 1}\right)+w\left(\frac{B_{n 2}}{B_{2}}-\frac{B_{n 1}}{B_{1}}\right)\right) \frac{\mathrm{d} \bar{f}_{2}\left(w_{\|}, w_{\perp}\right)}{\mathrm{d} w_{\|}}\right.$

$$
\left.\left.\left.+\left(\frac{w_{\perp}}{2 B_{2}}\left(B_{2}-B_{1}\right)+w\left(\frac{B_{t 2}}{B_{2}}-\frac{B_{t 1}}{B_{1}}\right)\right) \frac{\mathrm{d} \bar{f}_{2}\left(w_{\|}, w_{\perp}\right)}{\mathrm{d} w_{\perp}}\right]\right]\right\} .
$$

To simplify this expression we introduce the notations

$[[A]]=A_{2}-A_{1}$

$\{\{B\}\}=B_{1}+B_{2}$,

and Eq. (31) reduces to:

$$
\begin{aligned}
{\left[\left[\bar{f}\left(w_{\|}, w_{\perp}\right)\right]\right]=} & -\frac{1}{2}\left\{\left\{\frac{U_{n}}{U_{n}+w_{\|} \cos \alpha}\right.\right. \\
& \times\left(\left(\frac{B_{n}}{B}\left[\left[U_{n}\right]\right]+w\left[\left[\frac{B_{n}}{B}\right]\right]\right) \frac{\mathrm{d} \bar{f}\left(w_{\|}, w_{\perp}\right)}{\mathrm{d} w_{\|}}\right. \\
& \left.\left.\left.\times\left(\frac{w_{\perp}}{2 B}[[B]]+w\left[\left[\frac{B_{t}}{B}\right]\right]\right) \frac{\mathrm{d} \bar{f}\left(w_{\|}, w_{\perp}\right)}{\mathrm{d} w_{\perp}}\right)\right\}\right\} .
\end{aligned}
$$

This expression can be further simplified by introducing the notation

$$
\begin{aligned}
& C_{i}=\frac{U_{n i}}{U_{n i}+w_{\|} \frac{B_{n i}}{B_{i}}} \\
& A_{\| i}=\frac{B_{n i}}{B_{i}}\left[\left[U_{n}\right]\right]+w\left[\left[\frac{B_{n}}{B}\right]\right] \\
& A_{\perp i}=\frac{w_{\perp}}{2 B_{i}}[[B]]+w\left[\left[\frac{B_{t}}{B}\right]\right],
\end{aligned}
$$

and we finally get

$$
\begin{aligned}
{\left[\left[\bar{f}\left(w_{\|}, w_{\perp}\right)\right]\right]=- } & \frac{1}{2}\left\{\left\{C \cdot \left(A_{\|} \frac{\mathrm{d} \bar{f}\left(w_{\|}, w_{\perp}\right)}{\mathrm{d} w_{\|}}\right.\right.\right. \\
& \left.\left.\left.+A_{\perp} \frac{\mathrm{d} \bar{f}\left(w_{\|}, w_{\perp}\right)}{\mathrm{d} w_{\perp}}\right)\right\}\right\},
\end{aligned}
$$

which is a linear, partial, inhomogeneous differential equation of the first order for $\bar{f}_{2}$ in the variables $w_{\|}$and $w_{\perp}$.

\section{Results}

\subsection{Purely perpendicular shock}

First we investigate the reaction of the distribution function to a purely perpendicular shock, which leads to the conditions $B_{t 1}=B_{1}, B_{t 2}=B_{2}$, and $B_{n 1}=B_{n 2}=0$. In addition, from classical high Mach number MHD it follows that $U_{n 2}=U_{n 1} / s$ and $B_{2}=s B_{1}$. Using these results the MHD parameters introduced in Eqs. (35) to (37) reduce to

$C_{1,2}=1$

$A_{\|, 1,2}=0$

$A_{\perp, 1}=\frac{w_{\perp}}{2}(s-1)$

$A_{\perp, 2}=\frac{w_{\perp}}{2}\left(1-\frac{1}{s}\right)$.

Integrating Eq. (38) over velocity space then yields:

$$
\begin{aligned}
& n_{2}-n_{1}=-\frac{1}{4} \int \mathrm{d} \varphi w_{\perp} \mathrm{d} w_{\perp} \mathrm{d} w_{\|} w_{\perp}\left((s-1) \frac{\mathrm{d}}{\mathrm{d} w_{\perp}} \bar{f}_{1}\left(w_{\|}, w_{\perp}\right)\right. \\
&\left.+\left(1-\frac{1}{s}\right) \frac{\mathrm{d}}{\mathrm{d} w_{\perp}} \bar{f}_{2}\left(w_{\|}, w_{\perp}\right)\right) \\
& n_{2}-n_{1}= \frac{1}{2} \int \mathrm{d} \varphi w_{\perp} \mathrm{d} w_{\perp} \mathrm{d} w_{\|}\left((s-1) \bar{f}_{1}\left(w_{\|}, w_{\perp}\right)\right. \\
&\left.+\left(1-\frac{1}{s}\right) \bar{f}_{2}\left(w_{\|}, w_{\perp}\right)\right) \\
& n_{2}-n_{1}= \frac{1}{2}\left((s-1) n_{1}+\left(1-\frac{1}{s}\right) n_{2}\right) \\
& n_{2}-n_{1}= \frac{1}{2}\left((s-1) n_{1}+(s-1) n_{1}\right) \\
& n_{2}-n_{1}=(s-1) n_{1} \\
& n_{2}=s n_{1} .
\end{aligned}
$$

This is the classical MHD result, i.e., $n_{2}=s n_{1}$, which confirms that our kinetical ansatz and the resulting solution of the adequate Boltzmann equation is a valid description of a MHD shock.

\subsection{Purely parallel shock}

The other extreme situation in MHD shock theory is the parallel shock, which leads to the conditions $B_{t 1}=B_{t 2}=0$ and $B_{n 1}=$ $B_{n 2}=B$. In this case we obtain the following equations for the parameters:

$$
\begin{aligned}
& C_{1,2}=\frac{U_{n 1,2}}{U_{n 1,2}+w_{\|}} \\
& A_{\| 1}=A_{\| 2}=A_{\|}=U_{n 2}-U_{n 1}=U_{n 1}(1 / s-1) \\
& A_{\perp 1,2}=0 .
\end{aligned}
$$


Integrating Eq. (38) over velocity space then leads to

$$
\begin{aligned}
n_{2}= & n_{1}-A_{\|} \int \mathrm{d} \varphi w_{\perp} \mathrm{d} w_{\perp} \mathrm{d} w_{\|} \\
& \times\left(\frac{U_{n 1}}{U_{n 1}+w_{\|}} \frac{\mathrm{d}}{\mathrm{d} w_{\|}} \bar{f}_{1}\left(w_{\|}, w_{\perp}\right)+\frac{U_{n 2}}{U_{n 2}+w_{\|}} \frac{\mathrm{d}}{\mathrm{d} w_{\|}} \bar{f}_{2}\left(w_{\|}, w_{\perp}\right)\right) \\
= & n_{1}\left(1-g_{\|}\left(U_{1}, s\right)\right),
\end{aligned}
$$

where

$$
\begin{aligned}
& g_{\|}\left(U_{1}, s\right)=A_{\|} \int \mathrm{d} \varphi w_{\perp} \mathrm{d} w_{\perp} \mathrm{d} w_{\|} \\
& \quad \times\left(\frac{U_{n 1}}{U_{n 1}+w_{\|}} \frac{\mathrm{d}}{\mathrm{d} w_{\|}} \bar{f}_{1}\left(w_{\|}, w_{\perp}\right)+\frac{U_{n 2}}{U_{n 2}+w_{\|}} \frac{\mathrm{d}}{\mathrm{d} w_{\|}} \bar{f}_{2}\left(w_{\|}, w_{\perp}\right)\right) .
\end{aligned}
$$

To reproduce the classical MHD result, the condition

$g_{\|}\left(U_{1}, s\right)=n_{1}(1-s)$

needs to be fulfilled.

Solving the integral in Eq. (47) is complicated by the presence of the singularity in the form of $\left(U_{n}+w_{\|}\right)^{-1}$. In plasma physics, i.e., plasma wave dispersion theories, integrals of this form are common in the theory of wave damping and excitation, where they are solved by moving the singularity off the real axis and applying the residual theorem. In our case, this procedure allows us to apply Cauchys integral formula and we obtain

$$
\begin{aligned}
g_{\|}\left(U_{1}, s\right)= & \lim _{\epsilon \rightarrow 0} \frac{1}{i} \int w_{\perp} \mathrm{d} w_{\perp} \frac{\mathrm{d}}{\mathrm{d} w_{\|}}\left(\bar{f}_{1}\left(w_{\|}=U_{1}+i \epsilon, w_{\perp}\right)\right. \\
& \left.+\bar{f}_{2}\left(w_{\|}=U_{2}+i \epsilon, w_{\perp}\right)\right) .
\end{aligned}
$$

Using Eq. (48), we obtain the necessary conditions

$$
\begin{aligned}
& \operatorname{Re}\left(g_{\|}\left(U_{1}, s\right)\right)=0 \\
& \operatorname{Re} \lim _{\epsilon \rightarrow 0}\left(\int _ { 0 } ^ { \infty } w _ { \perp } \mathrm { d } w _ { \perp } \frac { \mathrm { d } } { \mathrm { d } w _ { \| } } \left(\bar{f}_{1}\left(w_{\|}=U_{1}+i \epsilon, w_{\perp}\right)\right.\right. \\
& \left.\left.\quad+\bar{f}_{2}\left(w_{\|}=U_{2}+i \epsilon, w_{\perp}\right)\right)\right)=0
\end{aligned}
$$

and

$$
\begin{aligned}
& \operatorname{Im}\left(g_{\|}\left(U_{1}, s\right)\right)=0 \\
& \operatorname{Im} \lim _{\epsilon \rightarrow 0}\left(\int _ { 0 } ^ { \infty } w _ { \perp } \mathrm { d } w _ { \perp } \frac { \mathrm { d } } { \mathrm { d } w _ { \| } } \left(\bar{f}_{1}\left(w_{\|}=U_{1}+i \epsilon, w_{\perp}\right)\right.\right. \\
& \left.\left.\quad+\bar{f}_{2}\left(w_{\|}=U_{2}+i \epsilon, w_{\perp}\right)\right)\right) \neq 0 .
\end{aligned}
$$

Since the downstream distribution function is an unknown function, it is in principle possible that Eq. (50) is valid. For Eq. (51), however, the situation is more clear. Since we are dealing with physical distribution functions, we get the additional condition $\operatorname{Im} \bar{f}_{i}=0$. The only possibility to produce a nonzero imaginary part of this expression uses the term $i \epsilon$, which is taken in the limit of $\epsilon \rightarrow 0$. Assuming that the upstream and downstream distribution functions are sufficiently smooth, we may pull the limit under the integral, and for a physical distribution function, the imaginary part will always vanish.

From this we conclude that for practically all physical distribution functions it is not possible to reproduce the MHD equations from a Boltzmann-kinetic treatment of the shock transition, as was developed in the section before. This is a direct result of the singularity at $w_{\|}=-U$. Considering the source of this expression (Eq. (17)), it may be interpreted as an effective velocity at which the individual particles with velocity $w_{\|}$cross the shock. Obviously, not only will some of these particles never make it past the shock, but they will cross it in the "wrong" direction, going from the downstream to the upstream region. This essentially splits up the system into (at least) two different streams moving in antiparallel directions.

\section{Higher order velocity moments}

\subsection{The downstream pressure anisotropy}

Despite the complications involved with a general solution of Eq. (38), we now investigate the downstream pressure anisotropy, which is of special interest because it cannot be predicted from classical MHD theories, not even if extended to nonscalar pressures such as those carried out by Erkaev et al. (2000). As shown by these authors, the system of shock relations resulting in this more complicated case can only be solved if the assumption is made that, according to derivations presented in the CGL-theory (Chew et al. 1956), the first and second adiabatic plasma invariants are conserved at the shock passage. However, even then the downstream pressure anisoptropy

$\lambda_{2}=\frac{p_{\perp, 2}}{p_{\|, 2}}$

must be kept as a free parameter of the solutions, since the system of shock relations is not completely closed. It is thus interesting now to see whether from the above kinetic approach one can obtain a fixed value for $\lambda_{2}$, which then would help to close the system of MHD shock relations.

In the case of a purely perpendicular shock, where the MHD mass flux conservation can always be fulfilled (see Sect. 5.1), no matter what the distribution function in detail looks like, we take Eq. (41) and apply cylindrical velocity space coordinates $\left(\mathrm{d}^{3} w=\mathrm{d} \varphi \mathrm{d} w_{\|} w_{\perp} \mathrm{d} w_{\perp}\right)$, which lead to the relations

$$
\begin{aligned}
& \mathrm{d} \varphi \mathrm{d} w_{\|} w_{\perp} \mathrm{d} w_{\perp} w_{\perp}^{n} \frac{\mathrm{d} \bar{f}_{1}}{\mathrm{~d} w_{\perp}} A_{\perp 1}=\mathrm{d} \varphi \mathrm{d} w_{\|} \mathrm{d} w_{\perp} \frac{n+1}{2} w_{\perp}^{n} \bar{f}_{1}(s-1) \\
& \mathrm{d} \varphi \mathrm{d} w_{\|} w_{\perp} \mathrm{d} w_{\perp} w_{\perp}^{n} \frac{\mathrm{d} \bar{f}_{2}}{\mathrm{~d} w_{\perp}} A_{\perp 2}=-\frac{\mathrm{d}}{\varphi} \mathrm{d} w_{\|} \mathrm{d} w_{\perp} \frac{n+1}{2} w_{\perp}^{n} \bar{f}_{2}(1-1 / s)
\end{aligned}
$$

where we have made use of $\mathrm{d}^{3} w=\mathrm{d} \varphi \mathrm{d} w_{\|} w_{\perp} \mathrm{d} w_{\perp}$ (i.e., cylindrical velocity space coordinates).

The partial pressures in the plasma wind system are defined as the second-order velocity moments,

$$
\begin{aligned}
& p_{\|, 2}=\left\langle\frac{m}{2} w_{\|}^{2}\right\rangle=\frac{m}{2} \int \mathrm{d}^{3} w w_{\|}^{2} \bar{f}_{2} \\
& p_{\perp, 2}=\left\langle\frac{m}{2} w_{\perp}^{2}\right\rangle=\frac{m}{2} \int \mathrm{d}^{3} w w_{\perp}^{2} \bar{f}_{2} .
\end{aligned}
$$

Instead of solving the differential equation, Eq. (38), and evaluating these above integrals, we use a more straightforward and easier way. We multiply Eq. (38) by $w_{\|}^{2}$ and $w_{\perp}^{2}$, integrate over velocity space, and obtain a much simpler system of algebraic equations for the downstream pressures as functions of the upstream pressures and the compression ratio.

The equations then reduce to

$$
\begin{aligned}
p_{\|, 2}-p_{\|, 1} & =\frac{m}{2} \frac{1}{2} \int \mathrm{d}^{3} w\left(\bar{f}_{1}(s-1)+\bar{f}_{2}(1-1 / s)\right) \cdot w_{\|}^{2} \\
& =\frac{1}{2}\left(p_{\|, 1}(s-1)+p_{\|, 2}(1-1 / s)\right)
\end{aligned}
$$




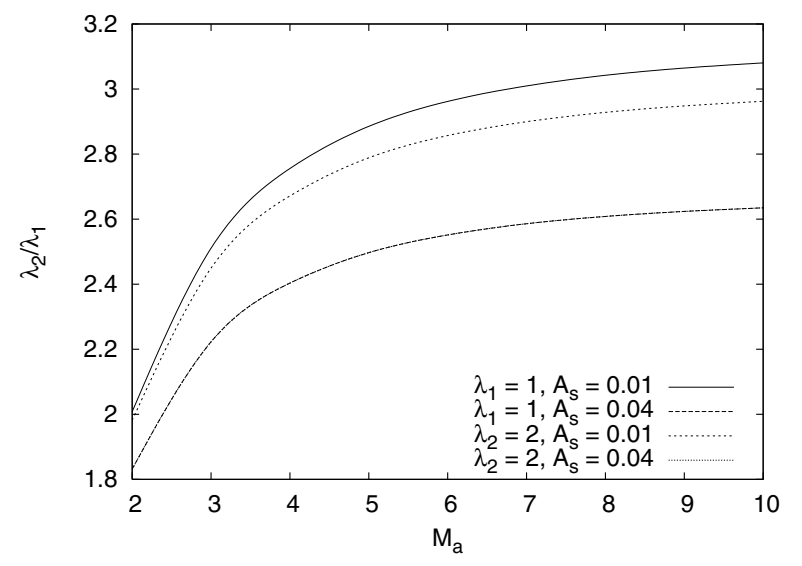

Fig. 1. Ratio of iterated downstream to upstream pressure anisotropy for a perpendicular shock as a function of the mach number $M_{\mathrm{a}}$ and for $A_{\mathrm{s}}=0.01$ and 0.04 (for a definition of $A_{\mathrm{s}}$ see Erkaev et al. 2000).

and

$$
\begin{aligned}
p_{\perp, 2}-p_{\perp, 1} & =\frac{m}{2} \int \mathrm{d}^{3} w\left(\bar{f}_{1}(s-1)+\bar{f}_{2}(1-1 / s)\right) \cdot w_{\perp}^{2} \\
& =p_{\perp, 1}(s-1)+p_{\perp, 2}(1-1 / s) .
\end{aligned}
$$

Solving these equations results in the pressures

$$
\begin{aligned}
& p_{\|, 2}=p_{\|, 1} s \\
& p_{\perp, 2}=p_{\perp, 1} s^{2}
\end{aligned}
$$

and the pressure anisotropy

$\lambda_{2}=\frac{p_{\perp, 2}}{p_{\|, 2}}=\frac{p_{\perp, 1} s^{2}}{p_{\|, 2} s}=\lambda_{1} s$

which interestingly means that the pressure anisotropy at the shock passage has changed and can now be fixed and introduced into the incomplete MHD solutions.

From this above result it is possible to draw two important conclusions: first, for a perpendicular shock, the downstream pressure anisotropy is a simple, monotonously growing function of the MHD compression ratio $s$. Furthermore, the downstream anisotropy will always be larger than the upstream one.

The second result is even more important, since the downstream pressures do not need the knowledge of the specific form of the ion velocity distribution function. As already mentioned, a pure MHD treatment of the shock is not able to predict the pressure anisotropy downstream. Now, however, we have the possibility of evaluating this expression numerically: we first select an initial $\lambda_{2,0}$, calulate the new $\lambda_{2,1}$ using $s\left(\lambda_{2,0}\right)$ and Eq. (61), and repeat this procedure until $\lambda_{2, i+1}\left(\lambda_{2, i}\right)=\lambda_{2, i}$. Since the MHD compression factor $s$ is, at least for a perpendicular shock, almost independent of $\lambda_{2}$ (Erkaev et al. 2000), this algorithm typically already converges after the first or second step. Iterated compression ratios are presented in Fig. 1, where we have demonstrated that the downstream pressure anisotropy depends strongly on the sonic mach parameter $A_{s}$ and somewhat less on the upstream pressure anisotropy $\lambda_{1}$ and the Alfven mach number $M_{A}$.

\subsection{MHD adiabatic invariants}

We now test if this above-derived result is consistent with the CGL-asumption of the conservation of the two adiabatic invariants as adopted in the MHD model by Chew et al. (1956) and Erkaev et al. (2000),

$$
\begin{aligned}
& \frac{\mathrm{d}}{\mathrm{d} t}\left(\frac{p_{\|} B^{2}}{\rho^{3}}\right)=0 \\
& \frac{\mathrm{d}}{\mathrm{d} t}\left(\frac{p_{\perp}}{\rho B}\right)=0 .
\end{aligned}
$$

We begin by transforming derivatives with respect to $t$ into those with respect to $s$ and obtain

$$
\begin{aligned}
& U_{n}(s) \frac{\mathrm{d}}{\mathrm{d} s}\left(\frac{p_{\|} B^{2}}{\rho^{3}}\right)=0 \\
& U_{n}(s) \frac{\mathrm{d}}{\mathrm{d} s}\left(\frac{p_{\perp}}{\rho B}\right)=0 .
\end{aligned}
$$

In the far upstream and downstream regions, where the MHD quantities are constant, these conditions are trivially fulfilled. To treat the transition between the upstream and downstream quantities, we apply our results from Sect. 3 and obtain

$\frac{1}{2}\left\{\left\{U_{n}(s)\right\}\right\}[[g(s)]]=0$,

which further simplifies itself to

$[[g(s)]]=0$.

For the adiabatic relations we then obtain

$$
\begin{aligned}
& \frac{p_{\| 2} B_{2}^{2}}{\rho_{2}^{3}}=\frac{p_{\| 1} B_{1}^{2}}{\rho_{1}^{3}} \\
& p_{\| 2}=s p_{\| 1} \\
& \frac{p_{\perp 2}}{B_{2} \rho_{2}}=\frac{p_{\perp 1}}{B_{1} \rho_{1}} \\
& p_{\perp 2}=s^{2} p_{\perp 1} .
\end{aligned}
$$

Obviously, both adiabatic conditions are valid, even though only one of these relations was explicitly used in our derivation of the Boltzmann equation.

From Sect. 3 and Eq. (28) it also follows that any adiabatic invariant of the form

$$
\frac{\mathrm{d}}{\mathrm{d} t} g(s)=0
$$

where $g(s)$ is an arbitrary function that is constant in the upstream and downstream regions automatically leads to an additional jump condition,

$[[g(s)]]=0$.

This result may prove very useful in obtaining a complete set of jump conditions in more complicated physical configurations.

\section{Conclusions and outlook}

We have developed a kinetic treatment of a pressure-anisotropic plasma crossing a classical MHD shock. To do this, we first derived the Boltzmann equation for such a plasma, taking the nonrelativistic limit from the general covariant form of the Boltzmann equation and adapting it to typical anisotropic MHD shock transitions. We have proven that for practically all physical situations the fine structure of the transition region does not influence the downstream quantities at all. 
Next, we investigated the shock transition in more detail for two extreme situations, the perpendicular shock where the magnetic field is orthogonal to the shock normal, and the parallel shock, where $B$ is parallel to the shock normal. We have proven that in the case of the perpendicular shock, the kinetic treatment of the shock is in perfect agreement with the MHD one, and it is possible to give an analytical expression for the downstream pressure anisotropy, closing the (incomplete) set of MHD equations (see, e.g. Erkaev et al. 2000) without any specific knowledge of the velocity distribution function.

For the parallel shock, we have proven that MHD is usually not an adequate description, which is a direct result from a singularity in the Boltzmann equation. We have physically interpreted this as a distribution in the bulk velocity system, where particles with different local velocities will also cross the shock at different timescales. This situation can be identified as (at least) two different plasma streams crossing the shock with two different velocities, both parallel and antiparallel to the shock normal. This effect will automatically lead to a two-stream instability leading to viscous-type dissipation, as was already often envisaged in the literature (e.g. Scholer et al. 2003), which, however, will be analyzed in more detail in a further publication.

While we have not yet solved the Boltzmann equation to obtain the downstream distribution function, we were already able to derive several results from the equation itself, including the lower order momenta of the downstream plasma. The full solution of the equation, as well as more detailed properties of this distribution function (e.g. higher downstream momenta, or the entropy change when crossing the shock) will be published in the near future. We have also recently begun investigating the generation of MHD waves and their coupling to the charged particles, including determining what kind of wave generation and radiation is possible from within the system. After finishing this, we intend to revisit the parallel shock (and, more generally, the inclined shock with an arbitrary angle between the magnetic field and the shock normal).

Our results suggest that a perpendicular shock is well described by simple analytical expressions, which do not depend on the fine structure of the shock at all. This in turn provides an excellent test criterium for numerical simulations aiming to describe more complex shock structures in plasmas. In addition, this relation and the formalism developed here should considerably simplify calculations related to less simple systems, e.g. where there are more than one particle population (see, e.g. Bzowski et al. 2006).

Finally, we were able to derive a simple relation which allows to turn any adiabatic invariant into an additional jump condition, which should allow us to study more complicated jump conditions.

Acknowledgements. We are grateful for financial support to the DFG within the frame of the DFG-Project Fa 97/31-1.

\section{References}

Baumjohann, W., \& Treumann, R. A. 1996, Basic space plasma physics (London: Imperial College Press), 176

Bzowski, M., Fahr, H.-J., \& Grzedzielski, S. 2006, ASTRA, 2, 1

Chalov, S. V., \& Fahr, H.-J. 1994, A\&A, 288, 973

Chalov, S. V., \& Fahr, H.-J. 1995, Planet. Space Sci., 43(8), 1035

Chalov, S. V., \& Fahr, H.-J. 1996, A\&A, 311, 317

Chew, G. F., Goldberger, M. L., \& Low, F. E. 1956, Proc. R. Soc. London A, 236, 112

Diver, D. A. 2001, A Plasma Formulary for Physics, Technology and Astrophysics (Hoboken, New Jersey: John Wiley)

Erkaev, N. V., Vogl, D. F., \& Biernat, H. K. 2000, J. Plasma Phys., 64, 561

Fahr, H.-J., \& Scherer, K. 2005, J. Geophys. Res., 110, A02103

Gombosi, T. I. 1998, Physics of the space Environment (New York: Cambridge University Press)

Hada, T., Onishi, M., Lembege, B., \& Savoinin, P. 2003, J. Geophys. Res., 108, 1233

Landau, L. D., \& Lifshitz, E. M. 1977, Lehrbuch der theoretischen Physik (Berlin: Akademiker-Verlag)

Lembege, B., Giacalone, J., Scholer, M., et al. 2004, Space Sci. Rev., 110, 161

Russell, C. T. 1995, Adv. Space Res., Vol. 15 (8/9), Physics of Collisionless Shocks

Scholer, M., Shinohara, I., \& Matsukiyo, S. 2003, J. Geophys. Res., 108, 1014

Scudder, J. D. 1995, Adv. Space Res., 15, 181

Serrin, J. 1959, Handbuch der Physik, Vol. VIII, Mathematical principles of classical fluid mechanics (Berlin: Springer Verlag), 125

Thomsen, M. F., Gosling, J. T., Bame, S. J., \& Mellot, M. M. 1985, J. Geophys. Res., 90, 137

Webb, G. M. 1985, ApJ, 296, 319

Wilkinson, W. P. 1991, J. Geophys. Res., 96, 17675

Zank, G. P. 1999, Space Sci. Rev., 89, 413

Zank, G. P., Webb, G. M., \& Donohue, D. J. 1993, ApJ, 406, 97

Zel'dovich, Y. B., \& Raizer, Y. P. 1966, Physics of shock waves and hightemperature hydrodynamic phenomena (New York: Academic Press), 75 